\title{
Chemotherapy Induced Peripheral Neuropathy - A Review
}

\author{
Ruchira Shrikant Ankar ${ }^{1}$, Seema Singh ${ }^{2}$ \\ ${ }^{1,2}$ Department of Medical Surgical Nursing, Smt. Radhikabai Meghe Memorial College of Nursing \\ Datta Meghe Institute of Medical Sciences, Sawangi (M) Wardha, Maharashtra, India.
}

\section{ABSTRACT}

\section{BACKGROUND}

Chemotherapy Induced Peripheral Neuropathy (CIPN) can be precipitated by many modern chemotherapeutic agents. CIPN affects the patient's quality of life not only physically, but also functionally, psychosocially, spiritually and affects the family as well. If severe acute syndrome develops during chemotherapy, doses of drugs should be reduced or even stopped. CIPN is a neurological side effect occurring during chemotherapy treatment in cancer patient depending on many factors such as age, dose strength, complete total drug dose received, period of therapy, use of more than two groups of neurotoxic agents, concomitant neuropathies (for example, diabetic neuropathy), genetic vulnerability, and alcoholism. Pathophysiology of CIPN is not clearly understood. The pathological processes by which chemotherapy drugs harm the nervous system structures cause CIPN. It depends on several factors or causes which include microtubule disruption, oxidative stress and mitochondrial break, changes in ion channel activity, myelin sheath and DNA damage, and neuro inflammation. Clinical manifestations vary from patient to patient. It is classified into three types such as sensory, motor and autonomic. Presently there are no standard guidelines for the assessment of CIPN. It is considered both objective evidence of neurological dysfunction and assessment of symptoms reported by the patient. Nurses can play a very important role in the early identification of CIPN in cancer patients which can help to plan and in modification of treatment. This will help patients to enjoy a better quality of life. This review aims to give an update regarding CIPN and nursing aspect.

\section{KEY WORDS}

Chemotherapy, Neuropathy, Risk Factors, Nursing
Corresponding Author: Ruchira Shrikant Ankar. Department of Medical Surgical Nursing, Smt. Radhikabai Meghe Memorial College of Nursing, Datta Meghe Institute of Medical Sciences, Sawangi (M) Wardha, Maharashtra, India.

E-mail: ruchira3110@gmail.com

DOI: $10.14260 /$ jemds/2020/689

How to Cite This Article: Ankar RS, Singh S. Chemotherapy induced peripheral neuropathy a review. J Evolution Med Dent Sci 2020;9(42):3147-3151, DOI: 10.14260/jemds/2020/689

Submission 18-07-2020,

Peer Review 11-09-2020,

Acceptance 18-09-2020,

Published 19-10-2020.

Copyright (C) 2020 Ruchira Shrikant Ankar et al. This is an open access article distributed under Creative Commons Attribution License [Attribution 4.0 International (CC BY 4.0)] 


\section{BACKGROUND}

Cancer worldwide is one of the leading causes increasing the mortality rate. ${ }^{1}$ In India, cancer is the second and fourth leading cause of death in urban and rural areas. ${ }^{2}$ Globally cancer survivors also increase as there is a significant advancement in cancer diagnosis and treatment modalities. ${ }^{3}$ Neurotoxicity from cancer treatment has been widely recognized. Chemotherapy may have significant effects on the central or peripheral nervous systems that can limit the course of treatment. Chemotherapy induced peripheral neuropathy (CIPN) can arise from many modern chemotherapeutic agents. If severe acute syndrome developed during chemotherapy; it causes a reduction in doses of drug or even stopping it. ${ }^{4}$ CIPN continues even though treatment completes and causes symptom burden which affects the patient's well-being.

Numerous factors such as age, maximum dose intensity, administration time of therapy, usage of more than two neurotoxic agents, simultaneous other neuropathies (for example, diabetic neuropathy), genetic predisposition, and alcohol abuse influence occurrence of CIPN. The onset, severity, characteristics, and duration of clinical manifestations of CIPN are highly variable. Neurotoxicity due to chemotherapy administration includes peripheral neuropathy which develops alteration in sense of temperature, tingling or burning in extremities. Also, in some patients, they develop changes in vision and hearing. These symptoms may increase as chemotherapy progresses. ${ }^{6}$ Also if patients having additional disease conditions such as diabetes, hypertension, hypothyroidism may worsen the degrees of CIPN. ${ }^{7}$ Currently, there are no preventive measures available for CIPN. Some drugs are used to reduced neuropathic pain such as tricyclic antidepressants, antiepileptic drugs, and adjuvant analgesics. ${ }^{8}$ CIPN affects the patient's quality of life not only physically, but also functionally, psychosocially, spiritually and also affects the family as well. ${ }^{9}$

Incidence of CIPN is chemotherapeutic drug-dependent varying from $19 \%$ to more than $85 \%{ }^{10}$ It is commonly found in platinum group of chemotherapy drugs (cisplatin, carboplatin., oxaliplatin) around $70-100 \%$, taxanes (paclitaxel, docetaxel)10 - $80 \%$, thalidomide and its analogues $20-60 \%$, and ixabepilone $60-65 \% .{ }^{9}$ Chemotherapy drugs when used in single large dose or maximum cumulative exposure occurs, it increased risk of lethal effect. This can change temperature sensation either temporarily / permanently in peripheral nerves with chronic pain and nerve damage. Some incidental studies of CIPN found that around 68 $\%$ observed in initial three months after exposure to neurotoxic chemotherapy drugs, $60 \%$ at three months and 30 $\%$ at and after 6 months. ${ }^{10}$ After completion or discontinuation of chemotherapy, patient may suffer with CIPN related symptoms such as pain or disturbances in sensations for months to years. Hence, patient may be recovering from cancer but suffering by chemotherapy induced neurological problems which affects their quality of life. ${ }^{11}$

\section{PATHOPHYSIOLOGY}

Pathophysiology of CIPN is not clearly understood. But it generally explains as symmetrical and bilateral axonopathy in nature in which cell bodies of dorsal root ganglia would be affected. Each chemotherapy drug has a different mechanism by which they induce their anti-mitotic effects. ${ }^{12}$ The pathological process of chemotherapy drugs harms the nervous system and develop CIPN. It depends on many factors or causes which include microtubule disturbances, oxidative stress, mitochondrial injury, alter functions of ion channel, harm to myelin sheath and DNA. ${ }^{13}$

Due to the effect of chemotherapy drugs, many changes develop in cells which may result in DNA damage, axonal changes or neuroinflammation. Drugs may act on mitochondria, membrane receptors and ion channels which change intracellular homeostasis, signalling and neurotransmission. ${ }^{14}$

\section{RISK FACTORS}

There are many studies conducted to identify predictors for CIPN. Patients with a history of diabetes mellitus, alcoholism or heredity of neuropathy are included as a risk for developing CIPN. Also, an indirect relationship in the pathogenesis of CIPN shown in patients with thyroid dysfunction, metabolic and infectious diseases like HIV, hepatitis B or C. Vitamin B1, B6 and B12 deficiencies and monocolonalgammopathy. ${ }^{6}$ Some drugs which are used in the treatment of cancer patient like metronidazole, misonidazole, sulfasalazine or phenytoin also reported in the involvement of some degree of peripheral neuropathy. 15

Some other predictors mention that patients with long duration of cancer or presently taking cancer therapy, older age, obesity or more body mass index, sleep disturbances with severe level, anxiety or depression are involved in CIPN.16 Patient with history of premature delivery, poor physical activities and higher cumulative dose of chemotherapy consider as predictors of CIPN. ${ }^{17}$

Genetic factors also identified as risk factors, even though there is a need for more research on potential contributory factors. Genome Wide Association Studies identified some single nucleotide polymorphisms possibly connected in the development of CIPN. Various protein functions have been recognized with axon outgrowth, sodium channels and neuronal apoptosis. ${ }^{18}$ Older age increase $6 \%$ more chance to develop CIPN. ${ }^{19}$ Also eight-time risk increase in a patient with a history of neuropathy for development of CIPN. ${ }^{17}$

\section{CLINICAL MANIFESTATIONS}

Clinical manifestations differ from patient to patient. It indicates "glove and stocking" distribution of symptoms as chemotherapy drugs are likely to target longer axons in the extremities. Symptoms classified in three types such as sensory, motor and autonomic. Major symptoms observed are sensory. ${ }^{20}$

After administration of chemotherapy, CIPN develops gradually and it progresses as treatment continues. ${ }^{21,14}$ Symptoms, of CIPN are very subjective and the most common sensory symptoms occur. Symptoms are likely to starts from the toes and move to feet, legs, hands, and arms. It is a progressive distal symmetrical. Patients reported numbness, 
tingling, pricking, burning, decreased or change in sensation or increase sensitivity that may be painful in extremities. ${ }^{22}$

These symptoms often are present concurrently and distress cancer patients by causing paraesthesia, altered function and damaging hearing and vision, etc. ${ }^{23}$ Cancer patients experience poor quality of life due to CIPN. It affects serious limitations in daily activities. ${ }^{24,25}$ Patient experiences CIPN symptoms like burning, numbness or pain in fingers or hands causes problems in the performance of daily activities like putting a button to a shirt, holding a pen, pencil or opening any container. Also, the same symptoms developing in toes causes the problem in walking, standing or climbing stairs. These are experiences that can develop depression, anxiety and disturbances in social relations also. ${ }^{26}$

\section{CLINICAL DIAGNOSIS}

Physicians are facing challenges to diagnose the CIPN and its management as no standard guidelines are available. For diagnosis of the patient both objective evidence of neurological dysfunction and symptoms reported by patient are considered. ${ }^{27}$ Even though there are issues in prevention and management, assessment for CIPN should be perform throughout the period of treatment. Assessment should involve diagnosis, functional disability and symptoms. ${ }^{28}$

There are grading scales available such as World Health Organization (WHO), Eastern Clinical Oncology Group (ECOG), National Cancer Information Center-Common Toxicity Criteria (NCIC-CTC), and Common Terminology Criteria for Adverse Events (CTCAE). ${ }^{29}$ Also some self-reporting tools are used to assess the functioning capacity of the patient. These are: Functional Assessment of Cancer Therapy / Gynaecologic Oncology Group Neurotoxicity (FACT / GOG-Ntx), Chemotherapy-Induced Peripheral Neuropathy Assessment Tool (CIPNAT),European Organization for Research \& Treatment in Cancer Quality of Life Quest-CIPN (EORTC QLQCIPN), Modified Total Neuropathy Score (mTNS), Total Neuropathy Score clinical version (TNSc) Total Neuropathy Score-reduced (TNSr). ${ }^{30,31}$

It is difficult to find out the level of physical limitation in patient's daily living activities. To confirm physical limitations, ask patient or family members regarding difficulties to perform daily activities such as unable to button a shirt, unsteady walk, fall during walking or writing problem. If such observations are noticed, doctor needs to modify, interrupt or discontinue chemotherapy.

\section{TREATMENT}

\section{Pharmacological Management}

Currently, there is lack in effective treatment regime which helps to prevent or lessen the CIPN. Although many pharmacological trials are conducted including acetyl-Lcarnitine, acetylcysteine, $\alpha$-lipoic acid, amifostine, amitriptyline, calcium / magnesium, carbamazepine, diethyldithiocarbamate, glutathione, gosha-jinki-gan, minocycline, nimodipine, omega-3 fatty acids, vitamin B and vitamin $\mathrm{E}$, none of them show effective results. One clinical trial conducted on duloxetine which shows positive evidence in treatment in CIPN. ${ }^{32,33}$ Also many clinical trials for CIPN fail to complete outcome guidelines given by IMMPACT. 34

Local applications of menthol only and also with baclofen, amitriptyline and ketamine has given effective results to reduce neuropathic pain and improvement in quality of life. Topical capsaicin $8 \%$ patch applications help to reduce neuropathic pain symptoms. This application also found to improve IENF density and improvement in patient condition. $35,36,37$

\section{Non-Pharmacological Management}

Non-pharmacological approaches include the use of vitamins, exercise and cold application. Patient education and good communication also essential in the management of CIPN.

Neuromodulation, transcutaneous electrical nerve stimulation, scramble therapy has shown improvement in CIPN symptoms but need clinical trials on large populations. ${ }^{38,39,40}$

A small sample study was conducted for the prevention of CIPN of cryotherapy with frozen socks, results show effectiveness, but evidence is limited. ${ }^{41}$

Regular exercises help to strengthen muscles and improve nerve functions, so it is essential to inform the patient and motivate them to perform daily some light exercise. Exercises help to improve coordination and neuromuscular functions; it is advised to practice with onset of symptoms of CIPN. ${ }^{42,43}$

To improve coordination, sensorimotor function and fine motor function the training should begin with the onset of manifest of CIPN at the latest.

\section{NURSING IMPLICATIONS}

Nurses can play very important role in early identification of CIPN in cancer patient which can help to plan and modify the treatment. This will help patients' to proceed to better quality of life. ${ }^{44}$ Chemotherapy induced nerve complications can be reduce by appropriate identification of symptoms which helps to reduce nerve injury. ${ }^{45}$ It is revealed that patients neglect symptoms of CIPN and often are unreported to doctors because of reasons like fear of discontinuing chemotherapy, doctors awareness that it is common side effect and there is no specific treatment available. ${ }^{26,46}$ Routinely nurses have to assess each cancer patients who are coming for chemotherapy with neurotoxic drugs for symptoms of peripheral neuropathy.

Nurses have to be aware that patient may not report or noticed CIPN symptoms as painful, so when doing assessment ask regarding any new numbness, tingling or any changes in sensation. If patient reported such symptoms, additional neurological assessment and fall risk assessment is necessary. ${ }^{47}$ At present to save patient's wellbeing nurses are recommended to give health education which includes identification of symptoms and lifestyle modification. 48,49,50

\section{Teaching Includes}

1. Orientated and make patient and their family members aware regarding clinical manifestations of CIPN and motivate them to report as early as they notice to their doctor. 
2. Teach patient and family members regarding importance of providing or keeping safe environment, because patient may lose sensations and may not able to identify injury to extremities.

3. Ask family members to apply non-skid surfaces on floors and tubs.

4. Keep well lighted room and illuminate stairs or entrance areas.

5. Encourage patient to observe feet daily for injury. Wear a closed-toed shoe which is properly fitted.

6. Avoid walking without footwear.

7. Avoid sitting with cross legs.

8. If sensations change, ask someone to check temperature of bath water or use water thermometer. Maintain temperature of water $120^{\circ} \mathrm{F}$ or below. Avoid very hot or cold water.

9. Protect extremities from cold by using gloves and socks, because patient is having risk to develop thermal injury.

10. Daily check skin for any cuts, abrasion or injury.

11. Explain symptoms of autonomic dysfunction which includes constipation, urinary retention, postural hypotension such can be managed by, before standing hang the legs then stand, use fiber rich diet and plenty of fluids.

12. Motivate the patient to perform regular light exercise such as walking which help to maintain physical activity and improve blood circulation.

13. Ask for quit smoking and alcohol

14. Explain to avoid activities which may cause stress on a nerve such as playing tennis, golf or typing.

Nurses should help to find out risk of safety problems in their surrounding and suggest changes, so it will prevent injuries or fall. ${ }^{9}$

\section{CONCLUSIONS}

Chemotherapy goals have changed from curative to continuation of treatment and better outcome with regard to quality of life. This increases the importance of vigilant practice during chemotherapy. Progression of treatment with chemotherapy increases risk of CIPN as there is till now no preventive treatment available. Nurses can play an important role in early identification of CIPN. During each visit of patient for chemotherapy, clinical assessment of upper and lower extremities is helpful to identify CIPN early. Education can help to identify risk factors in surrounding environment and modification in environment and activities help to maximize physical functioning of patients which help to achieve better outcome and quality of life.

Financial or other competing interests: None.

Disclosure forms provided by the authors are available with the full text of this article at jemds.com.

\section{REFERENCES}

[1] Ferlay J, Colombet M, Soerjomataram I, et al. Estimating the global cancer incidence and mortality in 2018:
GLOBOCAN sources and methods. Int J Cancer 2019;144(8):1941-53.

[2] Smith RD, Mallath MK. History of the growing burden of cancer in india: from antiquity to the 21 st century. J Glob Oncol 2019;5:1-15.

[3] Arnold M, Rutherford MJ, Bardot A, et al. Progress in cancer survival, mortality, and incidence in seven highincome countries 1995-2014 (ICBP SURVMARK-2): a population-based study. Lancet Oncol 2019;20(11):1493505.

[4] Stone JB, DeAngelis LM. Cancer treatment-induced neurotoxicity--a focus on newer treatments. Nat Rev Clin Oncol 2016;13(2):92-105.

[5] Khuresha R, Kaur R. Clinical \& genetic risk factors contribution to cipn (chemotherapy induced peripheral neuropathy): a systematic review \& meta-analysis. International Journal of Recent Scientific Research 2019;10(4):31873-82.

[6] Martin LGR, Silva MDP. Chemotherapy-induced peripheral neuropathy: a literature review. Einstein (São Paulo) 2011;9(4):538-44.

[7] Quasthoff S, Hartung HP. Chemotherapy-induced peripheral neuropathy. J Neurol 2002;249(1):9-17.

[8] Kaley T], Deangelis LM. Therapy of chemotherapyinduced peripheral neuropathy. $\mathrm{Br} \mathrm{J}$ Haematol 2009;145(1):3-14.

[9] Beijers A, Mols F, Dercksen W, et al. Chemotherapyinduced peripheral neuropathy and impact on quality of life 6 months after treatment with chemotherapy. J Community Support Oncol 2014;12(11):401-6.

[10] Fallon MT. Neuropathic pain in cancer. Br J Anaesth 2013;111(1):105-11.

[11] Flatters SJL, Dougherty PM, Colvin LA. Clinical and preclinical perspectives on chemotherapy-induced peripheral neuropathy (CIPN): a narrative review. Br J Anaesth 2017;119(4):737-49.

[12] Seretny M, Currie GL, Sena ES, et al. Incidence, prevalence, and predictors of chemotherapy-induced peripheral neuropathy: a systematic review and meta-analysis. Pain 2014;155(12):2461-70.

[13] Areti A, Yerra VG, Naidu V, et al. Oxidative stress and nerve damage: role in chemotherapy induced peripheral neuropathy. Redox Biol 2014;2:289-95.

[14] Starobova H, Vetter I. Pathophysiology of chemotherapyinduced peripheral neuropathy. Front Mol Neurosci 2017;10:174.

[15] Bao T, Basal C, Seluzicki C, et al. Long-term chemotherapyinduced peripheral neuropathy among breast cancer survivors: prevalence, risk factors, and fall risk. Breast Cancer Res Treat 2016;159(2):327-33.

[16] Ghoreishi Z, Keshavarz S, Jafarabadi MA, et al. Risk factors for paclitaxel-induced peripheral neuropathy in patients with breast cancer. BMC Cancer 2018;18(1):958.

[17] Miaskowski C, Mastick J, Paul SM, et al. Chemotherapyinduced neuropathy in cancer survivors. J Pain Symptom Manage 2017;54(2):204-218.e2.

[18] Wheeler HE, Gamazon ER, Wing C, et al. Integration of cell line and clinical trial genome-wide analyses supports a polygenic architecture of paclitaxel-induced sensory peripheral neuropathy. Clin Cancer Res 2013;19(2):4919. 
[19] Hershman DL, Till C, Wright JD, et al. Comorbidities and risk of chemotherapy-induced peripheral neuropathy among participants 65 years or older in southwest oncology group clinical trials. J Clin Oncol 2016;34(25):3014-22.

[20] Staff NP, Grisold A, Grisold W, et al. Chemotherapyinduced peripheral neuropathy: a current review. Ann Neurol 2017;81(6):772-81.

[21] Park SB, Goldstein D, Krishnan AV, et al. Chemotherapyinduced peripheral neurotoxicity: a critical analysis. CA Cancer J Clin 2013;63(6):419-37.

[22] da Simão DAS, Murad M, Martins C, et al. Chemotherapyinduced peripheral neuropathy: review for clinical practice. Rev Dor 2015;16(3):215-20.

[23] Wolf SL, Barton DL, Qin R, et al. The relationship between numbness, tingling, and shooting/burning pain in patients with chemotherapy-induced peripheral neuropathy (CIPN) as measured by the EORTC QLQCIPN20 instrument, N06CA. Support Care Cancer 2012;20(3):625-32.

[24] Tofthagen C, Donovan KA, Morgan MA, et al. Oxaliplatininduced peripheral neuropathy's effects on health-related quality of life of colorectal cancer survivors. Support Care Cancer 2013;21(12):3307-13.

[25] Bakitas MA. Background noise: the experience of chemotherapy-induced peripheral neuropathy. Nurs Res 2007;56(5):323-31.

[26] Alberti P. Chemotherapy-induced peripheral neurotoxicity - outcome measures: the issue. Expert Opin Drug Metab Toxicol 2017;13(3):241-3.

[27] Haryani H, Fetzer SJ, Wu CL, et al. Chemotherapy-induced peripheral neuropathy assessment tools: a systematic review. Oncol Nurs Forum 2017;44(3):E111-23.

[28] Alberti P, Rossi E, Cornblath DR, et al. Physician-assessed and patient-reported outcome measures in chemotherapy-induced sensory peripheral neurotoxicity: two sides of the same coin. Ann Oncol 2014;25(1):257-64.

[29] Gordon-Williams R, Farquhar-Smith P. Recent advances in understanding chemotherapy-induced peripheral neuropathy. F1000Res 2020;9:F1000.

[30] Yeo F, Ng CC, Loh KWJ, et al. Minimal clinically important difference of the EORTC QLQ-CIPN20 for worsening peripheral neuropathy in patients receiving neurotoxic chemotherapy. Support Care Cancer 2019;27(12):475362.

[31] Lavoie Smith EM, Haupt R, Kelly JP, et al. The content validity of a chemotherapy-induced peripheral neuropathy patient-reported outcome measure. Oncol Nurs Forum 2017;44(5):580-8.

[32] Beijers AJM, Jongen JLM, Vreugdenhil G. Chemotherapyinduced neurotoxicity: the value of neuroprotective strategies. Neth J Med 2012;70(1):18-25.

[33] Hershman DL, Lacchetti C, Dworkin RH, et al. Prevention and management of chemotherapy-induced peripheral neuropathy in survivors of adult cancers: American society of clinical oncology clinical practice guideline. J Clin Oncol 2014;32(18):1941-67.

[34] Dworkin RH, Turk DC, Farrar JT, et al. Core outcome measures for chronic pain clinical trials: IMMPACT recommendations. Pain 2005;113(1-2):9-19.

[35] Fallon MT, Storey DJ, Krishan A, et al. Cancer treatmentrelated neuropathic pain: proof of concept study with menthol--a TRPM8 agonist. Support Care Cancer 2015;23(9):2769-77.

[36] Barton DL, Wos EJ, Qin R, et al. A double-blind, placebocontrolled trial of a topical treatment for chemotherapyinduced peripheral neuropathy: NCCTG trial N06CA. Support Care Cancer 2011;19(6):833-41.

[37] Anand P, Elsafa E, Privitera R, et al. Rational treatment of chemotherapy-induced peripheral neuropathy with capsaicin 8\% patch: from pain relief towards disease modification. J Pain Res 2019;12:2039-52.

[38] Hofmeister M, Memedovich A, Brown S, et al. Effectiveness of neurostimulation technologies for the management of chronic pain: a systematic review. Neuromodulation Technol Neural Interface 2020;23(2):150-7.

[39] Abd-Elsayed A, Schiavoni N, Sachdeva H. Efficacy of spinal cord stimulators in treating peripheral neuropathy: a case series. J Clin Anesth 2016;28:74-7.

[40] Loprinzi C, Le-Rademacher JG, Majithia N, et al. Scrambler therapy for chemotherapy neuropathy: a randomized phase II pilot trial. Support Care Cancer 2020;28(3):118397.

[41] Hanai A, Ishiguro H, Sozu T, et al. Effects of cryotherapy on objective and subjective symptoms of paclitaxelinduced neuropathy: prospective self-controlled trial. JNCI J Natl Cancer Inst. 2018;110(2):141-8.

[42] Kleckner IR, Kamen C, Gewandter JS, et al. Effects of exercise during chemotherapy on chemotherapy-induced peripheral neuropathy: a multicenter, randomized controlled trial. Support Care Cancer 2018;26(4):101928.

[43] Jordan K, Feyer P, Höller U, et al. Supportive treatments for patients with cancer. Dtsch Ärztebl Int 2017;114(2728):481-7.

[44] Al-Atiyyat N, Banifawaz AZ. Oncology nurses' knowledge, practice, and confidence toward chemotherapy-induced peripheral neuropathy in Jordan. Saudi Med J 2018;39(11):1158-63.

[45] Taverner T. Neuropathic pain: an overview. Br J Neurosci Nurs 2014;10(3).

[46] Tofthagen C, Visovsky C, Hopgood R. Chemotherapyinduced peripheral neuropathy: an algorithm to guide nursing management. Clin J Oncol Nurs 2013;17(2):13844.

[47] Managing chemotherapy-induced peripheral neuropathy. Oncology Nursing News https://www.oncnursingnews.com/publications/oncolo gy-nurse/2013/june-2013/managing-chemotherapyinduced-peripheral-neuropathy

[48] Chemotherapy-induced peripheral neuropathy treatment guidelines June 2019. https://rmpartners.nhs.uk/wpcontent/uploads/2019/07/RM-Partners-CIPN-

Treatment-Guidelines-June-2019.pdf

[49] Nursing best practice guide peripheral neuropathy. https://academy.myeloma.org.uk/wpcontent/uploads/sites/2/2018/01/Myeloma-AcademyBest-Practice-Peripheral-Neuropathy-2017.pdf

[50] Managing peripheral neuropathy. https://www.cancer.org/treatment/treatments-andside-effects/physical-side-effects/peripheralneuropathy/managing-peripheral-neuropathy.html 